\title{
Interpretación a partir del análisis cinético de los resultados del ensayo acelerado (IRAM 1674) para la reacción álcali-silice pavimentos:
}

INTERPRETATION FROM THE KINETIC ANALYSIS OF THE ACCELERATED TEST RESULTS (IRAM 1674) FOR THE ALKALI-SILICA REACTION.

Ing. Patricia E. Vila, MSc. (Estudiante de doctorado) Asistente del Instituto de Estructuras y Transporte, Universidad de la República, Uruguay.

pvila@fing.edu.uy
Ing. María Noel Pereyra, PhD.

Profesor del Instituto de Estructuras y Transporte, Universidad de la República, Uruguay.

mpereyra@fing.edu.uy

Fecha de recepción: 04 JULIO 2017 / Fecha de aprobación: 28 SETIEMBRE 2017

Índices y Bases de Datos:

\section{lationdex ucRIndex}

\section{REDIB O Dialnet DOA}

revistas.ucr.ac.cr/index.php/materiales

(c) lanamme.ucr.ac.cr

@ metodosymateriales.lanamme@ucr.ac.cr
Políticas de Uso:

\section{(C) $\odot \Theta \Theta$}

Revista Métodos y Materiales por LanammeUCR se distribuye bajo: Licencia Creative Commons Atribución-NoComercial-SinDerivar 4.0 Internacional. ISSN impreso: 2215-342X. ISSN electrónico: 2215-4558 


\title{
Interpretación a partir del análisis
} cinético de los resultados del ensayo acelerado (IRAM 1674) para la
reacción álcali-sílice pavimentos.

\author{
INTERPRETATION FROM THE KINETIC ANALYSIS OF THE ACCELERATED TEST RESULTS (IRAM 1674) \\ FOR THE ALKALI-SILICA REACTION.
}

\section{Ing. Patricia E. Vila, MSc. (Estudiante de doctorado)}

Asistente del Instituto de Estructuras y Transporte, Universidad de la República, Uruguay.

pvila@fing.edu.uy
Ing. María Noel Pereyra, PhD.

Profesor del Instituto de Estructuras y Transporte, Universidad de la República, Uruguay.

mpereyra@fing.edu.uy

Fecha de recepción: 04 JULIO 2017 / Fecha de aprobación: 28 SETIEMBRE 2017

\section{RESUMEN}

En este trabajo se presentan los resultados de expansión según el ensayo acelerado en barras de mortero (IRAM 1674) inmersas durante 14 días en una solución agresiva y el análisis cinético según las recomendaciones RILEM AAR-2 para el estudio de la potencialidad deletérea del agregado frente a la reacción álcali-sílice. El objetivo principal del trabajo es contribuir a la interpretación de los resultados del ensayo acelerado, a partir del análisis del crecimiento de la expansión con el tiempo de inmersión (análisis cinético) otorgándole así una mayor confiabilidad al ensayo. El análisis cinético consiste en ajustar por mínimos cuadrados a los datos experimentales de expansión, un modelo matemático conocido (modelo KAMJ), de donde surgen los parámetros Ink y M.

Los parámetros cinéticos Ink y M están asociados al desarrollo de la reacción. Si bien en la bibliografía se propone un valor límite para Ink de -6 (valores menores, agregados no reactivos y valores mayores, agregados deletéreos), en el análisis de los datos experimentales se observó que valores bajos de este parámetro podrían estar asociados a un crecimiento no asintótico de la expansión y, de esta manera, presentar un comportamiento deletéreo. Se considera que el coeficiente Ink/M es un buen indicador para evaluar el comportamiento del agregado. Sin embargo, para poder definir un valor límite para el coeficiente Ink/M, resulta necesario establecer una correlación con los ensayos en prismas de hormigón de larga duración (1año o más) o de la experiencia en campo del comportamiento del agregado.

PALABRAS CLAVE: Reacción Álcali-Sílice. Ensayo Acelerado. Análisis Cinético.

\section{ABSTRACT}

The expansion of the accelerated test in mortar bars test immersed for 14 days in an aggressive solution (IRAM 1674) as well as the kinetic analysis according to RILEM AAR-2 recommendations for the study of the potencial deleterious of the aggregate in the alkali-silica reaction are presented in this work. The main objective is to contribute to the interpretation of the test results based on the analysis of the growth in expansion with the time of immersion (kinetic analysis) and in this way, get more reliability. The kinetic analysis consists in adjusting by least squares fit of the experimental data of expansion to a known mathematical model (model KAMJ), where the parameters Ink and $M$ are obtained from.

The kinetic parameters Ink and $M$ are associated with the behavior of the reaction. Although a limit value for Ink of -6 is proposed in the literature (lower values are non-reactive aggregates and higher values are deleterious aggregate), from the analysis of the experimental data it was observed that low values of this parameter could be associated with not asymptotic growth of expansion and, therefore, the aggregates presents a deleterious behavior. The Ink/M coefficient is considered a good indicator to evaluate the behavior of the aggregate. However, in order to define a limit value for Ink/M coefficient, it is necessary to establish a correlation with the tests on long-term concrete prism (1 year or more) or the field experience of the aggregate behavior.

KEYWORDS: Alkali-Silica Reaction. Accelerated Test. Kinetic Analysis. 


\section{INTRODUCCIÓN}

La reacción álcali-sílice (RAS) es una reacción interna en el hormigón que se da entre ciertos minerales considerados "reactivos" de los agregados (diferentes formas de cuarzo con sílice reactiva, ftanita, chert, calcedonia, tridimita, cristobalita, ópalo y vidrio volcánico) y los álcalis provenientes principalmente del cemento portland (identificados como iones de sodio y de potasio). Para su desarrollo es necesario de un medio alcalino habitual en la solución presente en la porosidad del hormigón. A consecuencia y en presencia de humedad, los productos de la reacción se expanden, generando agrietamiento y reduciendo significativamente la vida útil de la estructura afectada. Además, producen manchas por lixiviados en la superficie del hormigón (Nixon y Sims, 2016).

Por este motivo se han realizado grandes esfuerzos para desarrollar métodos de ensayo que permitan evaluar la reactividad del agregado y el grado deletéreo de la reacción. Hoy en día existen varios ensayos normalizados reconocidos internacionalmente, como lo son: el ensayo acelerado de la barra de mortero (ASTM C1260, 2014; IRAM 1674, 1997; ABNT NBR 15577-4, 2009; AENOR UNE 146508 EX, 1999), el ensayo semi-acelerado en prisma de hormigón (IRAM 1700, 2013; Nixon y Sims, 2016) y el ensayo en prisma de hormigón a largo plazo (1 año o más de duración)(ASTM C1293, 2015; IRAM 1700, 2013). El criterio para definir la reactividad del agregado surge del resultado de expansión que se obtiene luego de haber sometido las barras de mortero $\mathrm{u}$ hormigón a un ambiente agresivo por un período de tiempo.

El ensayo acelerado de expansión en barra de mortero, si bien tiene la ventaja de que los resultados se obtienen a corto plazo (a los 16 días de fabricadas las barras), tiene sus limitaciones. Por un lado, por su carácter acelerado, puede suceder que agregados considerados reactivos según este ensayo, luego en servicio no lo sean. Por otro lado, es cuestionada la interpretación que surge del ensayo para ciertos tipos de agregados (denominados de reacción lenta). De los ensayos existentes, el ensayo en prismas de hormigón a largo plazo es el que mejor representa el comportamiento del agregado en servicio (Falcone y Milanesi, 2012). Sin embargo, por los plazos que esto implica, el ensayo acelerado en la barra de mortero sigue siendo utilizado para caracterizar a los agregados en cuanto a su reactividad potencialfrente a la RAS.

Recientemente, el Comité Técnico 219-ACS del RILEM (comité que trabajó en el desarrollo de los diferentes métodos de ensayo de laboratorio para la evaluación de la RAS y en la confección de especificaciones para minimizar el daño ocasionado en estructuras hidráulicas de hormigón), incluyó entre sus recomendaciones, un nuevo método para la interpretación del resultado del ensayo acelerado de la barra de mortero, basado en el análisis cinético de la evolución de la expansión con el tiempo de ensayo (Nixony Sims, 2016). Johnston y Fournier (2000) y Johnston et al (2004) fueron los primeros en introducir este método de interpretación que surge de aplicar el modelo matemático conocido como KolmogorovAvrami-Mehl-Johnson (abreviado modelo KAMJ).

Los resultados de expansión con el tiempo de ensayo presentan un comportamiento según una función sigmoide (denominada curva en forma de "s"), donde al principio se presenta un importante incremento de la expansión en poco tiempo, pero luego de pasar por un punto de inflexión, la expansión decrece progresivamente hacia un valor asintótico (Stokes, 2006). El modelo matemático de KAMJ describe la nucleación y la cinética de la reacción de crecimiento y trasformación de fases en la etapa de crecimiento asintótico de la reacción. Tal modelo relaciona el porcentaje de expansión (Et) de manera exponencial con el tiempo $t$ (en días) como se muestra en la Ec.1:

$$
\mathrm{Et}(\%)=1+\mathrm{E}_{\mathrm{t} 1}(\%)-\mathrm{e}^{-\mathrm{k}(\mathrm{t}-\mathrm{t} 1)^{\mathrm{M}}}
$$

Donde $\mathrm{E}_{\mathrm{t} 1}$ es el grado de reacción en el tiempo $\mathrm{t}_{1}$ (en días) cuando la nucleación y crecimiento se convierten en dominantes y $\mathrm{k}$ es una constante de velocidad que combina los efectos de nucleación, crecimiento multidimensional, geometría de los productos de la reacción y de difusión. M es un término exponencial relacionado con el crecimiento y forma de la nucleación. Este modelo puede ser aplicado al desarrollo de la expansión tanto en barras de mortero como en prismas de hormigón, siendo una importante herramienta para realizar proyecciones o estimaciones del comportamiento del material a diferentes tiempos de ensayo o edades de expansión (Johnston y Fournier, 2000; Johnston et al 2004; Stokes, 2006).

Para los agregados que presentan una expansión intermedia en el ensayo acelerado de la barra de mortero, la norma del ensayo indica continuar hasta los 28 días. Mediante la aplicación del modelo cinético, el presente trabajo analiza un criterio de evaluación que permita tener una mayor confiabilidad en la interpretación de los resultados de expansión hasta los 14 días de ensayo. A su vez, en agregados que presentan un comportamiento asintótico de la reacción, el análisis permitiría la estimación de la expansión a edades mayores a 14 días. 


\section{OBJETIVO}

A partir de los resultados de expansión hasta los 14 días de inmersión del ensayo acelerado de la barra de mortero (IRAM 1674) y considerando el criterio cinético recomendado por el RILEM AAR-2 para la interpretación de los resultados, se busca establecer los parámetros cinéticos que permitan una mayor confiabilidad del criterio utilizado para la evaluación del agregado frente a la RAS.

Si bien el RILEM AAR-2 recomienda que se realice el análisis cinético de la expansión, no indica valores límites en los parámetros que surgen del análisis. El presente trabajo pretende contribuir, aportando resultados de estos coeficientes que permitan, en trabajos futuros definir criterios de reactividad a partir de estos coeficientes.

\section{METODOLOGÍA}

\subsection{Materiales y equipo}

Para el estudio fueron utilizados diferentes tipos de agregados todos ellos considerados aptos para hormigón (según UNIT
1050, 2005). De un total de 15 muestras, 6 fueron de agregado grueso (GD, GC, GM, GPCB, GMC, GFM), 6 de arena gruesa (AGL16, AGHA, AGB, AGR1, AGCP, AGCT), 2 de arena terciada (ATB, ATV) y 1 dearena fina (AFC). El agregadogrueso, salvo el GPCB (pedregullo), fue piedra partida (material que proviene de un proceso de trituración realizado en la cantera de extracción para obtener un tamaño de partícula apto para hormigón) y las arenas todas de origen natural. El criterio para definir las arenas como "arena gruesa" y "arena fina" surge de su distribución granulométrica. Las arenas gruesas presentan tamaños de grano mayores, en Uruguay generalmente la mayor proporción en estas arenas queda retenida en los tamices de mayor abertura (entre 2,36 $\mathrm{mm}$ y $0,30 \mathrm{~mm}$ ). Las arenas finas habitualmente presentan tamaño de grano comprendido ente $0,30 \mathrm{~mm}$ y 0,15 mm. Ambos tipos de arena deben ser mezcladas para conformar una distribución granulométrica adecuada para su uso en hormigón. Sin embargo, la "arena terciada" presenta una distribución granulométrica adecuada para su uso en hormigón. En la Tabla 1 se indica la identificación de cada muestra, procedencia, descripción resumida, eventual presencia de minerales reactivos según IRAM 1531 (2016) e IRAM 1512 (1998) y reactividad en el ensayo acelerado de la barra de mortero según IRAM 1674 (1997).

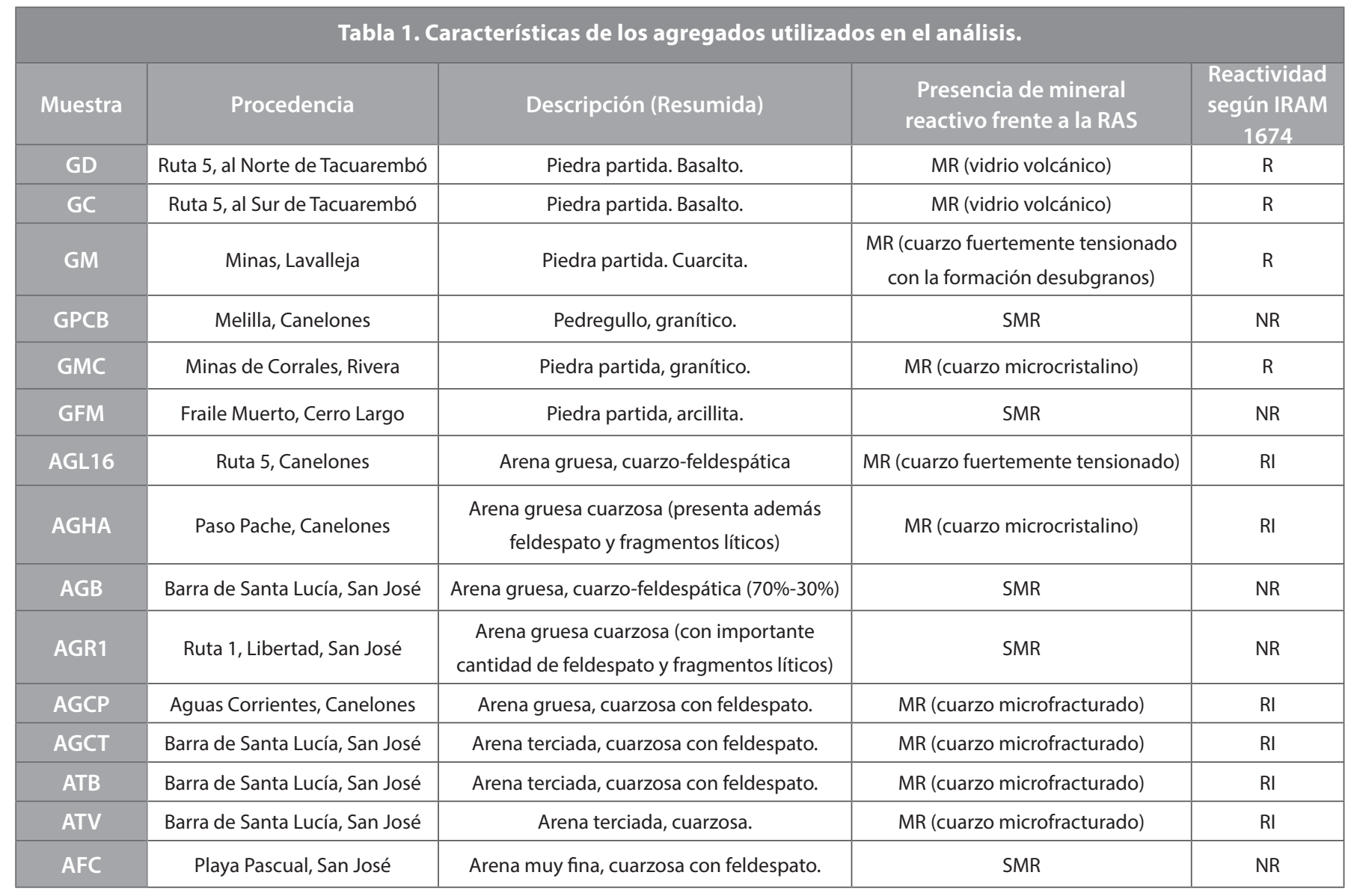

Nota: MR: presencia de mineral reactivo, SMR: no presenta mineral reactivo. R: reactivo, NR: no reactivo, RI: zona intermedia de expansión (entre 0,1\%0,2\%) en el método acelerado de la barra de mortero según IRAM 1674. 
Para la detección de los minerales reactivos las muestras de agregado fueron analizadas al microscopio mediante la confección de láminas delgadas. Como minerales reactivos, los agregados gruesos contenían vidriovolcánico y cuarzo fuertemente tensionado y microcristalino, salvo las muestras de GPCB y GFM donde no se observaron minerales reactivos. Las arenas presentaron diferentes tipos de cuarzo; tensionado, microfracturado y microcristalino, salvo las muestras de AGB, AGR1 y AFC donde no se observó la presencia de minerales reactivos.

Para el ensayo acelerado de la barra de mortero se fabricaron dos morteros, elaborándose tres barras a partir de cada uno por muestra de agregado. El agregado grueso se debió triturar para obtener los tamaños requeridos por la norma del ensayo (IRAM 1674) y las arenas fueron utilizadas en su tamaño natural(Tabla 2). En todos los casos se utilizó un tamaño menor a 4,75mm y se descartó el pasante tamiz 0,15 mm.

\begin{tabular}{|c|c|c|c|c|c|}
\hline \multicolumn{5}{|c|}{$\begin{array}{c}\text { Tabla 2. Distribución granulométrica de los agregados } \\
\text { utilizados en el ensayo acelerado. }\end{array}$} \\
\cline { 2 - 6 } & \multicolumn{5}{|c|}{$\%$ retenido en cada tamiz } \\
$\begin{array}{c}\text { Tipo de } \\
\text { agregado }\end{array}$ & $2,36 \mathrm{~mm}$ & $1,18 \mathrm{~mm}$ & $0,60 \mathrm{~mm}$ & $0,30 \mathrm{~mm}$ & $0,15 \mathrm{~mm}$ \\
\hline $\begin{array}{c}\text { Agr. } \\
\text { Grueso }\end{array}$ & $10 \%$ & $25 \%$ & $25 \%$ & $25 \%$ & $15 \%$ \\
\hline $\begin{array}{c}\text { Arena } \\
\text { Terciada }\end{array}$ & $10 \%$ & $25 \%$ & $25 \%$ & $25 \%$ & $15 \%$ \\
\hline $\begin{array}{c}\text { Arena } \\
\text { Gruesa }\end{array}$ & $10 \%$ & $25 \%$ & $25 \%$ & $40 \%$ & - \\
\hline $\begin{array}{c}\text { Arena } \\
\text { Fina }\end{array}$ & - & - & - & $85 \%$ & $15 \%$ \\
\hline
\end{tabular}

El cemento utilizado para la fabricación de los morteros es un cemento portland normal identificado como CPN 40 por la Norma UNIT 20 (2017) (cemento que contiene únicamente clinker como componente principal, sin adiciones y de uso general en el hormigón). Las muestras de CPN utilizadas presentaron un contenido de álcalis equivalente $\left(\mathrm{Na}_{2} \mathrm{O}_{\mathrm{eq}}\right)$ comprendido entre 1,16 \% a 1,29\%, una expansión en autoclave promedio de $0,11 \%$ y una superficie específica (Blaine) promedio de $330 \mathrm{~m}^{2} / \mathrm{kg}$.

Para las lecturas de expansión se utilizó un comparador vertical de recorrido máximo $(15 \pm 0,001) \mathrm{mm}$ y una barra de referencia de $300 \mathrm{~mm}$. Todas las barras fueron fabricadas de $25 \mathrm{~mm} 25 \mathrm{mmx} 285 \mathrm{~mm}$, con una longitud nominal libre entre pernos de medición de $(250 \pm 2) \mathrm{mm}$.

\subsection{Metodología del análisis}

Para cada una de las barras y durante el período de inmersión de 14 días en la solución agresiva de $1 \mathrm{~N} \mathrm{NaOH}$ a $(80 \pm 5)^{\circ} \mathrm{C}$ se registró la longitud inicial (identificada como $\mathrm{L}_{0}$ ), la longitud a tiempos de ensayo intermedios $\mathrm{L}_{\mathrm{ti}} \mathrm{y}$ la longitud al final del período de inmersión $\mathrm{L}_{14}$ para cada una de las barras. La expansión a la edad de inmersión $\mathrm{E}_{\mathrm{t}}$ se calculó como la diferencia entre la lectura a esa edad $\mathrm{L}_{\mathrm{ti}}$ y la lectura inicial $\mathrm{L}_{0}$, expresado como un porcentaje del largo nominal de la barra $\mathrm{L}_{\mathrm{n}}(250 \mathrm{~mm})$ según Ec. 2:

$$
\mathrm{Et}(\%)=100 *\left(\mathrm{~L}_{\mathrm{ti}}-\mathrm{L}_{0}\right) / \mathrm{L}_{\mathrm{n}}
$$

El resultado de expansión por muestra de agregado surge como el promedio de expansión de todas las barras ensayadas. Se calculó el coeficiente de variación por muestra para verificar la precisión de las medidas cumpla con límites indicados por la ASTM C1260 (2014).

Para analizar la evolución de la reacción RAS con el tiempo de inmersión t se determinó la curva de crecimiento de la expansión relativo a la expansión total $\mathrm{E}_{14}$ (en \%) y se realizó el análisis cinético recomendado por la RILEM AAR-2 (Nixon y Sims, 2016) aplicando el modelo matemático de KAMJ (Ec.1). Realizando el doble logaritmo natural a ambos lados de la Ec. 1 y haciendo un cambio de variable se obtiene el modelo de comportamiento lineal (Ec. 3) en las variables "y" y " $x$ ", según Ec. 4 y Ec. 5 (Islam y Ghafoori, 2013).

$$
y=M^{*} x+\operatorname{Ink}
$$

Donde:

$$
\begin{aligned}
& y=\operatorname{In}\left(\operatorname{In}\left(\frac{1}{1+E_{t 1}-E_{t}}\right)\right) \\
& x=\operatorname{In}\left(t-t_{1}\right)
\end{aligned}
$$

Para obtener los parámetros cinéticos $M$ y lnk de la Ec. 3 se realizó el ajuste lineal por mínimos cuadrados a los datos experimentales. El tiempo de inducción $t_{1}$ fue adoptado como la primera lectura intermedia durante los 14 días de inmersión (tiempo comprendido entre 1 y 4 días).

Dado que la cantidad de lecturas intermedias $\mathrm{L}_{\mathrm{ti}}$ no fue la misma para todas las muestras (respetándose la cantidad mínima de tres según la norma del ensayo), la cantidad de resultados de expansión a edades intermedias no fue la misma para todas las muestras. Por lo tanto, al aplicar el modelo de ajuste la cantidad de datos experimentales $\left(t, E_{t}\right)$ no fue la misma para todas las muestras. A su vez, la primera lectura intermedia (en t1) es una constante ingresada cuando se realiza el cambio de variable y no se considera como dato para el ajuste del modelo. 


\section{RESULTADOS Y DISCUSIÓN}

\subsection{Evolución de la expansión y aplicación del modelo cinético}

Para una mejor visualización de los resultados, las muestras fueron identificadas por color según el criterio de reactividad de la norma del ensayo (IRAM 1674) que surge de la evaluación de la expansión a los 14 días de inmersión $\mathrm{E}_{14}$ (rojo: $\mathrm{E}_{14}>0,2 \%$; naranja: $\mathrm{E}_{14}$ entre $0,1 \%$ y $0,2 \%$; verde: $\mathrm{E}_{14}<0,1 \%$ ). Primero se analizó la evolución de la expansión con la edad de ensayo relativo a $\mathrm{E}_{14}$ (Figura 1) y luego se determinaron los coeficientes del modelo cinético (Ec. 1) mediante el cambio de variables (Ec. 4 y Ec. 5) y ajuste por mínimos cuadrados a los datos experimentales según Ec. 3 (Figura 2 y Tabla 3).

Las muestras con $\mathrm{E}_{14}>0,2 \%$ (GD, GC, GMy GMC) presentaron una evolución de la expansión con el tiempo de ensayo diferente entre ellas. Las muestras GD, GC y GM presentaron una evolución similar, con un crecimiento más rápido que el resto, mientras que la muestra GMC presentó un crecimiento más lento, con un comportamiento menos asintótico (Figura 1). Entre las muestras con $\mathrm{E}_{14}$ comprendido entre 0,1 \% y 0,2\% (AGL16, AGHA, ATB, ATV, AGCP y AGCT) y las muestras con $\mathrm{E}_{14}<0,1 \%$ (AGB, AGR1, AFC, GPCB y GFM), presentaron una evolución similar de la reacción, salvo la muestra GFM que a tiempos intermedios de ensayo presentó un crecimiento más rápido que el resto.

Cuando se realiza el cambio de variables según Ec. 4 y Ec. 5 y se aplica el modelo de correlación lineal (Ec. 3) para cada muestra, se observó que los valores de la ordenada " $y$ " aumentaron en función del tiempo de ensayo (Figura 2), obteniéndose en todos los casos un buen ajuste a los datos experimentales $\left(\mathrm{R}^{2}>\right.$ 0,97 , en algunas muestras se tienen sólo tres resultados para el ajuste, lo que genera un $\mathrm{R}^{2}$ muy cercano a la unidad) (Tabla 3). Las muestras con una mayor magnitud de la expansión $\left(\mathrm{E}_{14}\right)$ presentaron una regresión lineal (recta) más próxima al eje de las abscisas (Figura 2). Entre las muestras que presentaron $\mathrm{E}_{14}>$ 0.2 \% se observó una importante diferencia en la muestra GM (valores de "y" mayores que el resto) y en la muestra de GMC que presentó una mayor pendiente $(M)$ y un menor valor de intercepción con la ordenada (lnk). En las muestras con $\mathrm{E}_{14}$ entre $0,1 \%$ y $0,2 \%$ se observó una mayor pendiente (M) y menor valor de lnk para la muestra AGCP. Entre las muestras con $\mathrm{E}_{14}<0,1 \%$,GPCB y GFM presentaron una pendiente $\mathrm{M}$ menor (Figura 2 y Tabla 3 ).

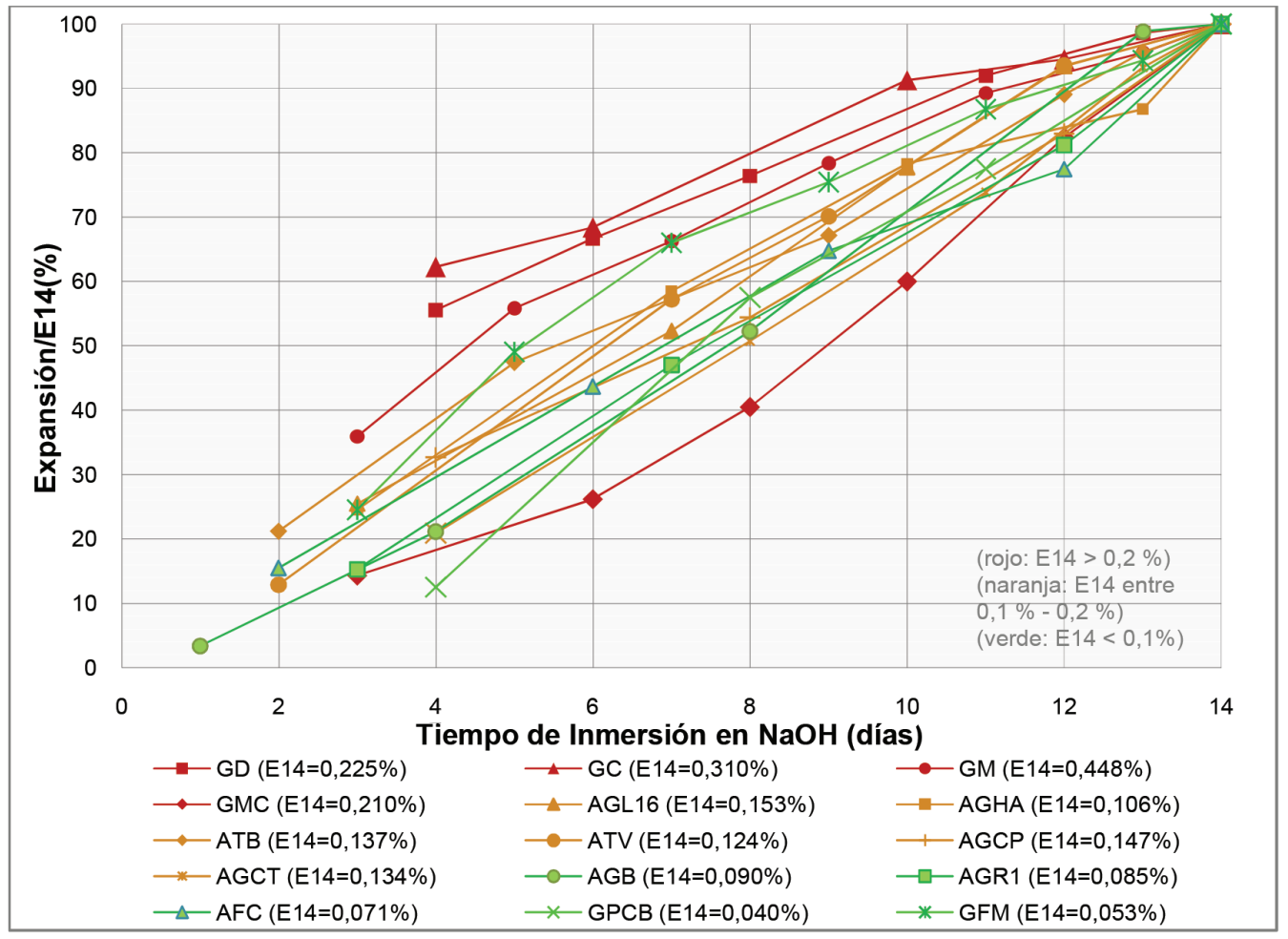

Figura 1. Gráfico de evolución de la expansión con el tiempo de ensayo. 


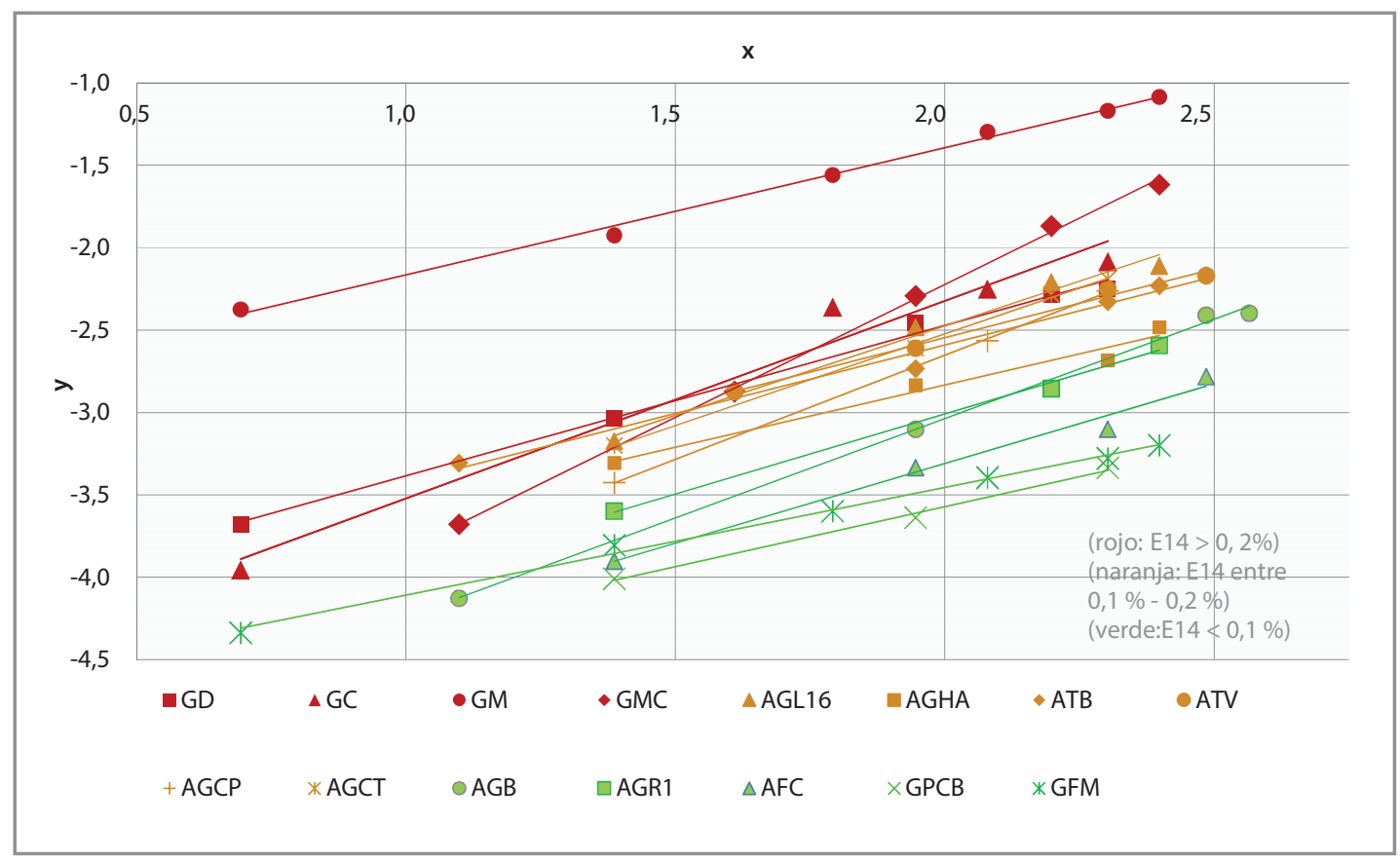

Figura 2. Gráfico del modelo ajustado a los datos experimentales.

Para una misma edad de ensayo, una expansión mayor resulta en una mayor diferencia entre $\mathrm{E}_{\mathrm{t} 1} \mathrm{y} \mathrm{E}_{\mathrm{t}}$, por lo tanto, un mayor valor de la variable "y" (Ec. 4). Por este motivo los modelos de regresión lineal se "desplazan hacia la abscisa". Este comportamiento se observa principalmente en el agregado GM (Figura 2) donde, si bien presentó un crecimiento asintótico similar a GD y GC, presentó valores de "y" bastante mayores como consecuencia de una elevada expansión $\left(\mathrm{E}_{14}=0,448 \%\right)$.

La mayor pendiente de GMC (Figura 2) está asociada a un crecimiento más lento de la reacción (Figura 1), observándose un crecimiento menos asintótico que el resto de las muestras.

\begin{tabular}{|c|c|c|c|c|}
\hline \multicolumn{5}{|c|}{$\begin{array}{c}\text { Tabla 3. Resultados de expansión y parámetros } \\
\text { del modelo para las muestras analizadas. }\end{array}$} \\
\hline Muestra & E14 & M & Ink & $\mathbf{R}^{2}$ \\
\hline GD & 0,225 & 0,879 & $-4,255$ & 0,986 \\
\hline GC & 0,310 & 1,199 & $-4,721$ & 0,971 \\
\hline GM & 0,448 & 0,770 & $-2,935$ & 0,996 \\
\hline GMC & 0,210 & 1,612 & $-5,447$ & 0,999 \\
\hline AGL16 & 0,153 & 1,086 & $-4,646$ & 0,984 \\
\hline AGHA & 0,106 & 0,753 & $-4,340$ & 0,972 \\
\hline ATB & 0,137 & 0,831 & $-4,253$ & 0,987 \\
\hline ATV & 0,124 & 0,837 & $-4,220$ & 0,991 \\
\hline AGCP & 0,147 & 1,264 & $-5,181$ & 1,000 \\
\hline AGCT & 0,134 & 1,113 & $-4,749$ & 0,998 \\
\hline AGB & 0,090 & 1,206 & $-5,450$ & 0,998 \\
\hline AGR1 & 0,085 & 0,971 & $-4,952$ & 0,996 \\
\hline AFC & 0,071 & 0,968 & $-5,244$ & 0,983 \\
\hline GPCB & 0,040 & 0,729 & $-5,029$ & 0,996 \\
\hline GFM & 0,053 & 0,653 & $-4,761$ & 0,996 \\
\hline
\end{tabular}


Analizando conjuntamente la evolución de la reacción (Figura 1) y los parámetros que surgen de la aplicación del modelo cinético (Figura 2 y Tabla 3), se observa que el coeficiente $M$ está asociado al crecimiento de la reacción; para muestras de agregado con una magnitud de expansión similar, cuanto más rápido se desarrolló la reacción, la pendiente $\mathrm{M}$ de la recta de ajuste fue menor. Por otro lado, el valor del parámetro lnk (intercepción de la recta con el eje de la ordenada) no solo depende del crecimiento relativo de la expansión a distintos tiempos de ensayo, sino también de la magnitud de la expansión. Puede observarse que muestras con valores similares de $M$, podrían presentar diferentes valores de lnk (como es el caso de GD y GM). Por lo tanto, las muestras que presentaron un valor bajo de $\mathrm{M}$ (menor pendiente) y un valor bajo de lnk, son aquellas que presentaron valores menores de expansión y un crecimiento más rápido de la reacción (comportamiento más asintótico).

Lo anterior muestra que ambos parámetros son necesarios para la caracterización del agregado, al igual que Johnston y Fournier (2000) quienes concluyeron que estos parámetros están asociados a la cinética de la reacción ( $k$ es una constante de velocidad que combina los efectos de nucleación, crecimiento multidimensional y la geometría de los productos de la reacción y difusión y $\mathrm{M}$ está relacionado con el crecimiento y la forma de la nucleación).

Si bien los parámetros cinéticos pueden ser calculados solamente con tres puntos (como ser con $\mathrm{t}_{1}, \mathrm{t}_{\mathrm{i}} \mathrm{yt}_{14}$ ), al no contar con un valor de referencia de $M$, son importantes las lecturas intermedias que permiten visualizar el comportamiento de la curva. Sólo en el caso de un comportamiento no asintótico de la expansión hasta los 14 días (debido a un prolongado tiempo de inducción de la reacción) podría ser necesario continuar el ensayo a edades superiores para mejorar su confiabilidad (como es el caso de la muestra GMC).

Las diferencias en la expansión $\left(E_{14}\right)$ y en la evolución durante el tiempo de ensayo dependen principalmente de la presencia y características del mineral (o minerales) reactivo en el agregado. La utilización del agregado triturado o en su estado natural también podría afectar su comportamiento. Los agregados GD, GC procedieron de la trituración de una roca volcánica (basalto) en la cual el mineral reactivo fue el vidrio volcánico. El agregado GM provino de la trituración de una roca cuarcita que presentó cuarzo fuertemente tensionado, con una importante formación de subgranos de sílice reactiva (Tabla 1). Ambos minerales generaron que los agregados GD, GC y GM presentaran una elevada expansión y un crecimiento más rápido que el resto (RILEM AAR 1, Nixon y Sims, 2016). La muestra de GMC provino de la trituración de una roca granítica cuyo mineral reactivo fue el cuarzo microcristalino, generando un crecimiento más lento de la reacción. Los agregados que presentaron un $\mathrm{E}_{14}$ comprendido entre $0,1 \%$ y $0,2 \%$ fueron arenas, utilizadas en su estado natural (sin triturar) y en presencia de cuarzo con un grado de reactividad intermedia. Entre los agregados que presentaron $\mathrm{E}_{14}<0,1 \%$ (AGB, AGR1, AFC, GPCB y GFM), GPCB y GFM presentaron un $\mathrm{M}$ menor, atribuyéndolo a la trituración de sus partículas para confeccionar las muestras del ensayo (dentro del grupo, el resto de los agregados fueron arenas usadas en su estado natural).

\subsection{Análisis de los parámetros cinéticos}

Se graficaron ambos parámetros cinéticos ( $\mathrm{M}$ vs lnk) permitiendo visualizar mejor su comportamiento (Figura 3). En el mismo gráfico se agregaron valores de los coeficientes extraídos de la bibliografía y zonas de reactividad según experiencia de campo (Johnston y otros, 2004; Bornstein y otros, 2015). Como tendencia general se observó que los coeficientes que surgen de los datos experimentales propios presentaron valores similares a algunos de los reportados en la bibliográfica, lo que permite reforzar (o extender) el análisis de los mismos a la experiencia de campo reportada.

Se observa que valores mayores de lnk junto con valores bajos de $\mathrm{M}$ dan agregados con elevada reactividad (como es el caso de GM). Valores mayores de lnk están asociados a valores de expansión altos y por lo tanto considerados expansivos. Sin embargo, para valores menores de lnk el grado de reactividad del agregado dependerá del parámetro $\mathrm{M}$, por lo tanto, valores bajos de lnk junto con valores bajos de $\mathrm{M}$ indican un mejor comportamiento del agregado.

Dado que el comportamiento cinético de la reacción está relacionado con ambos parámetros cinéticos ( $\mathrm{M}$ y lnk), se calculó el cociente lnk/M. Valores menores de este cociente, asociados a un menor lnk junto con un menor valor de $\mathrm{M}$, sugieren agregados con un mejor comportamiento frente a la reacción. Por el contrario, valores altos de este parámetro indican agregados deletéreos. Este comportamiento se puede observar en el gráfico de la Figura 4, donde se incluyen además de los datos experimentales propios, datos de la bibliografía. 


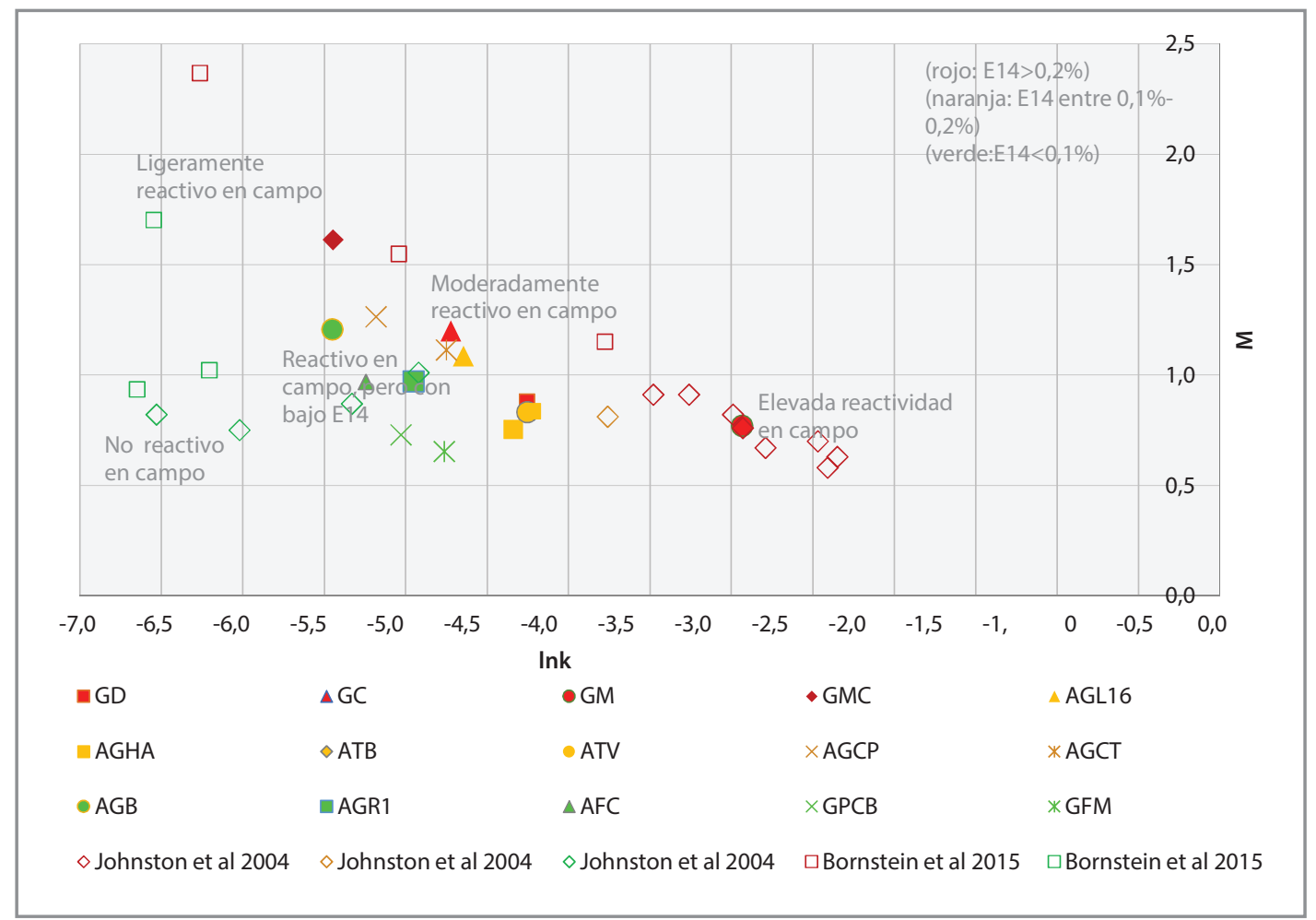

Figura 3. Gráfico de M vs Ink.

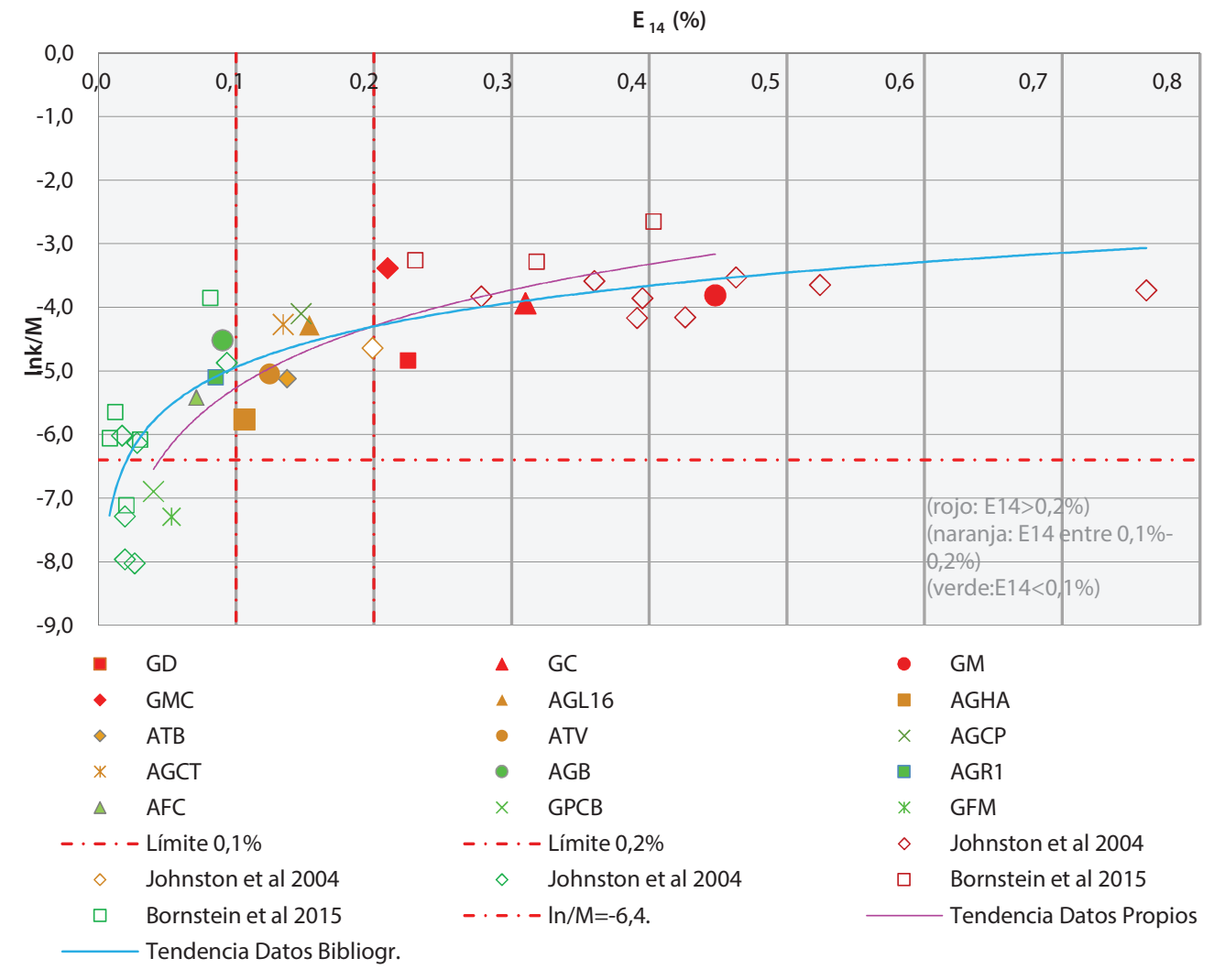

Figura 4.Gráfico de Lnk/M vs $\mathrm{E}_{14^{\circ}}$ 
Johnston et al (2004) realizaron un estudio de correlación entre los parámetros cinéticos que surgen del ajuste por el modelo de KAMJ de la expansión del ensayo acelerado de la barra de mortero (según la ASTM C1260) y el agrietamiento obtenido en prismas de hormigón expuestos al ambiente. Los resultados de este estudio se incluyen en los gráficos de las Figuras 3 y 4 . Estos autores recomiendan adoptar un valor de lnk < -6 para un comportamiento inocuo del agregado en campo. En otro trabajo (no incluido en las figuras) Johnston y Fournier (2000)concluyeron que las barras analizadas en el ensayo acelerado con un lnk $>-6$, presentaron agregados con un comportamiento deletéreo en prismas de hormigón sometidas al ensayo durante un año, lnk $<-6$, inocuos y próximos a -6 un comportamiento intermedio. Si bien en los datos experimentales propios no se presentaron muestras con lnk <-6, en el trabajo de Bornsteinet al (2015) se observó algún agregado considerado como reactivo con un valor de lnk $<-6$ (Figura 3).

Islam y Ghafoori (2013) realizaron el estudio cinético comparando los resultados de expansión en barras de mortero y en prismas de hormigón hasta la edad de 13 semanas de ensayo (ambos sumergidos en una solución agresiva de $1 \mathrm{~N}$ $\mathrm{NaOH}$ a $80^{\circ} \mathrm{C}$ ). Al igual que en el presente trabajo, adoptaron el coeficiente lnk/M para concluir sobre el grado de reactividad del agregado y a partir de la evaluación del agrietamiento en los prismas de hormigón, proponen el límite de lnk/M = -6,4 (Figura 4). De los datos experimentales sólo los agregados GPCB y GFM estarían por debajo de este límite. Sin embargo, dado que este límite surgió a partir de la evaluación en prismas de hormigón expuestos en un ambiente más agresivo que el de su comportamiento en campo (en servicio), podría considerarse como un valor conservador. Por lo tanto y según recomendaciones obtenidas de la bibliografía (Ideker et al 2012), será necesario hacer una comparación entre los resultados de laboratorio y el comportamiento del agregado en campo (o de correlación con prismas de hormigón, pero en un ambiente menos agresivo para tener un valor más exacto del límite de reactividad que el que surge de los ensayos acelerados en laboratorio).

El tiempo adoptado como t1 varió entre 1 y 4 días (primer día de lectura de expansión en cada muestra). Sin embargo, para tener una mejor comparación entre los parámetros cinéticos, debería adoptarse el mismo valor para todas las muestras (por ejemplo, tres días como recomienda la RILEM AAR-2).

\section{CONCLUSIONES}

La evolución de la expansión hasta los 14 días de inmersión en el ensayo acelerado de la barra de mortero (según IRAM 1674) para el estudio de la RAS, se pudo correlacionar con el modelo matemático recomendado por la RILEM AAR-2. El cálculo de los parámetros cinéticos $\operatorname{lnk}$ y $\mathrm{M}$ son un buen indicador para el análisis de la reacción. Si bien en la bibliografía se propone un valor límite para lnk de -6 (valores menores, agregados no reactivos, valores mayores, agregados deletéreos), del análisis de los datos experimentales se observó que valores bajos de este parámetro podrían estar asociados a un crecimiento no asintótico de la expansión y, por lo tanto, presentar un comportamiento igualmente deletéreo. El presente trabajo propone que el criterio de reactividad del agregado surja del cociente lnk/M.

Si bien se considera que el coeficiente $\operatorname{lnk} / \mathrm{M}$ es un buen indicador para evaluar el comportamiento del agregado, para poder definir un valor límite para el coeficiente lnk/M, resulta necesario establecer una correlación con los ensayos en prismas de hormigón de larga duración (1 año o más) o de la experiencia en campo del comportamiento del agregado.

Si bien, hoy en día, para los agregados que presentan una expansión intermedia en el ensayo acelerado de la barra de mortero, la norma del ensayo (IRAM 1674) indica continuar hasta los 28 días, al aplicar el criterio que surge del análisis cinético y en los casos de un comportamiento asintótico durante los 14 días de inmersión, no será necesario continuar por un período mayor de tiempo. Esto se ve reflejado en el parámetro $\mathrm{M}$, sólo valores altos de este parámetro podrían estar asociados a un comportamiento no asintótico del crecimiento de la reacción, dejando de ser válido el modelo cinético de KAMJ . En estos casos se debería continuar con el ensayo a edades posteriores. Sin embargo, es necesario una mayor cantidad de datos experimentales y de análisis de la curva de evolución de la reacción con el tiempo de ensayo que permitan cuantificar el límite para el coeficiente $\mathrm{M}$.

\section{AGRADECIMIENTOS}

Se agradece al Instituto de Estructuras y Transporte (IET) de la Facultad de Ingeniaría (FIng-UdelaR) por el equipamiento y lugar físico necesario para la realización de los ensayos, así como a las empresas que suministraron las muestras de agregado y cemento. Un agradecimiento al geólogo $\mathrm{S}$. Huelmo por su colaboración en el análisis al microscopio de las diferentes muestras y a los laboratoristas B. Epifanio y M Piriz por su colaboración en la preparación y ensayos de laboratorio. Se agradece a los revisores de la revista Métodos y Materiales que con sus aportes enriquecieron este trabajo. 


\section{BIBLIOGRAFÍA}

ABNT NBR 15577-4 (2009), Agregados - Reatividade álcali-agregado. Parte 4: Determição de expansão em barras de argamassa pelo método acelerado. Associação Brasilera de Normas Técnicas, Brasil.

- AENOR UNE 146508 EX (1999), Ensayos de áridos. Determinación de la reactividad potencial álcali-sílice y álcali-silicato de los áridos. Método acelerado en probetas de mortero. Asociación Española de Normalización y Certificación, España.

- ASTM C1260 (2014), Standard Test Method for Potential Alkali Reactivity of Aggregates (Mortar-Bar Method), ASTM International, West Conshohocken, PA, 2014, www.astm.org.

- ASTM C1293-08b (2015), Standard Test Method for Determination of Length Change of Concrete Due to Alkali-Silica Reaction, ASTM International, West Conshohocken, PA, 2015, www.astm.org.

Bronstein, B., Hocking, D., Bacolod, J. Sirivivatnanon., V. (2015). The use of reaction kinetics in classifying alkali silica reactivity potential of aggregates, en Proceddings of the concrete 2015 conference: 27th biennial national conference of the concrete institute of Australia in conjunction with the 69th RILEM week conference, 2015, pp. 704-711. ISBN: 978-1-943847-70-9.

- Falcone D., Milanesi, C., Método acelerado del prisma de hormigón. Evaluación de la RAS con agregados de diversas regiones de la Argentina, en el V Congreso Internacional y $19^{\circ}$ Reunión Técnica de la AATH, Bahía Blanca, Argentina, 2012.

Ideker, J., Bentivegna, A., Folliard, K., y Juenger, M., (2012). Do current laboratory test methods accurately predict alkali-silica reactivity? ACI MaterialsJournal, 109(4), 395-402.

- IRAM 1674(1997). Agregados. Determinación de la Reactividad Alcalina Potencial Método Acelerado de la Barra de Mortero,
Instituto Argentino de Normalización y Certificación, Argentina.

- IRAM 1700 (2013). Agregados. Método para la determinación del cambio de largo en prismas de hormigón, debido a la reacción álcali-agregado. Instituto Argentino de Normalización y Certificación, Argentina.

- Isam, M., Ghafoori, N., 2013, Evaluation of Alkali-Silica Reactivity Using Aggregate Geology, Expansion Limits of Mortar Bars and Concrete Prisms, and Kinetic Model. Journal of Materials Science Research, Vol. 2, No 2, 103-116.

Johnston, D. y Fournier, B., A kinetic-based method for interpreting accelerated mortar bar test (ASTM C1260) data, en Alkali-Aggregate Reaction, Proceedings of the 11th International Conference, Québec, Canada, 2000, 355-364.

Johnston, D., Stokes, D., Fournier, B.,Surfahl, R. Oy otros (2000), Kinetic characteristics of ASTM C1260 testing and ASR-induced concrete damge, en 12th International Conference on Alkali-Aggregate Reaction in Concrete, Beijung, China, 2004.

- Nixon, P y Sims, I. (2016), RILEM Recommendations for the Prevention of Damage by Alkali-Aggregate Reactions in New Concrete Structures, State-of-the-Art Report of the RILEM Technical Committee 219-ACS.

- Stokes, D., Concerning the Use of Expansion Data from AST Testing, en Marc-Andre Berube Symposium on Alkali-Aggregate Reactivity in Concrete, ACI International Conference on Recent Advances in Concrete Technology, Québec, Canada, 2006.

- UNIT 1050 (2005). Proyecto y ejecución de estructuras de hormigón en masa o armado. Instituto Uruguayo De Normas Técnicas, (Montevideo, Uruguay), p. 288.

- UNIT 20 (2017), Cemento pórtland para uso general. Definiciones y requisitos. Instituto Uruguayo De Normas Técnicas, (Montevideo, Uruguay), p. 13. 